\title{
Fermi Edge Singularities in the Mesoscopic Regime: I. Anderson Orthogonality Catastrophe
}

\author{
Martina Hentschel, ${ }^{1,2}$ Denis Ullmo, ,, and Harold U. Baranger ${ }^{1}$ \\ ${ }^{1}$ Duke University, Department of Physics, Box 90305, Durham, NC 27708-0305 \\ ${ }^{2}$ Institut für Theoretische Physik, Universität Regensburg, 93040 Regensburg, Germany
}

(Dated: September 11, 2018)

\begin{abstract}
For generic mesoscopic systems like quantum dots or nanoparticles, we study the Anderson orthogonality catastrophe (AOC) and Fermi edge singularities in photoabsorption spectra in a series of two papers. In the present paper we focus on $\mathrm{AOC}$ for a finite number of particles in discrete energy levels where, in contrast to the bulk situation, AOC is not complete. Moreover, fluctuations characteristic for mesoscopic systems lead to a broad distribution of AOC ground state overlaps. The fluctuations originate dominantly in the levels around the Fermi energy, and we derive an analytic expression for the probability distribution of AOC overlaps in the limit of strong perturbations. We address the formation of a bound state and its importance for symmetries between the overlap distributions for attractive and repulsive potentials. Our results are based on a random matrix model for the chaotic conduction electrons that are subject to a rank one perturbation corresponding, e.g., to the localized core hole generated in the photoabsorption process.
\end{abstract}

PACS numbers: 73.21.-b,78.70.Dm,05.45.Mt,78.67.-n

\section{INTRODUCTION}

Among the simplest many-body problems one can consider is a Fermi sea of electrons interacting with a static impurity. Such an impurity is, for example, suddenly formed when a core or valence electron is excited above the Fermi energy by an incident photon in a bulk-metal photoabsorption or photoluminescence experiment. Naively, one would expect a sharp onset of the absorption cross section at threshold with a signal proportional to the density of states in the conduction band above the Fermi energy. In experiments, deviations from this behavior were found in the form of peaked or rounded edges both in metals $\frac{1}{\underline{1}}$ and semiconductors ${ }^{2}$ These so-called Fermi edge singularities (FES) are a clear indication of the importance of many-body processes that have been receiving enduring interest.

For (clean) macroscopic systems, an essentially complete understanding of the mechanisms causing the Fermi edge singularities has been obtained by the late eighties ${ }^{3.4 .5 .6 .7}$ In particular, these works have emphasized the role of two competing effects. The first one is the Anderson orthogonality catastrophe (AOC), which is a property of the overlap between many-body wavefunctions. However, in x-ray absorption and photoabsorption processes, besides AOC there is another many-body response of the conduction electrons to the

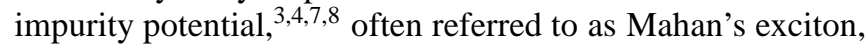
Mahan's enhancement, or the Mahan-Nozières-DeDominicis (MND) contribution. It counteracts the effect of AOC in situations where the dipole selections rules are fulfilled, and causes the photoabsorption cross section to diverge at the threshold.

In the present series of two papers we are interested in these many-body effects in mesoscopic systems,, 9.10 .11 such as quantum dots or nanoparticles, and in particular in the changes with respect to the macroscopic (or bulk) case due to the confined geometry. In this first paper, we focus on the orthogonality catastrophe mechanism for a finite system. We will more particularly consider the case of a contact (rank one), but not necessarily small, perturbation, with the localized perturbation presented by the core hole left behind in photoabsorption just being one example. In the second paper, that will be referred to as II in the following, we will discuss in detail Fermi edge singularities and mesoscopic photoabsorption spectra that depend not only on AOC but crucially on the MND contribution.

In a bulk-metal $\mathrm{x}$-ray absorption experiment, the conduction electrons respond to the abrupt, non-adiabatic appearance of a core hole generated upon photoexcitation of an inner shell electron into the conduction band by adjusting their single particle energies and wavefunctions slightly. Since the core hole represents an effectively positive charge the scattering potential will be attractive, and the energy levels will be lowered. Although the overlap of the respective single particle states before and after the perturbation is very close to one, the many-body ground state overlap $\Delta$ tends to zero in the thermodynamic limit, $\Delta \propto M^{-\epsilon}$ with $M$ the number of conduction electrons and the parameter $\epsilon>0$. This effect, first noted by Anderson in 1967,,$\frac{12}{,}$ is the Anderson orthogonality catastrophe. As a result of AOC the cross section $A(\omega)$ for the absorption of photons with energy $\omega$ will be power-law suppressed near threshold, explaining the above-mentioned Fermi edge singularity in the form of a rounded edge.

AOC plays an important role in all processes where a finite range scattering potential suddenly arises or changes. It is also prominent in the Mössbauer effect where AOC enables the recoilless emission or absorption of $\gamma$-rays by the whole crystal, but not by a single nucleus 13 Another example is Kondo physics, except that in that case the impurity is dynamic, rather than static as considered here.

Turning from macroscopic to mesoscopic systems opens a new venue to the study of AOC. To start with, the confined geometry will naturally induce new behavior associated with the mesoscopic regime, among which are the lack of translational or rotational invariance, the existence of interference effects, and the related mesoscopic fluctuations ${ }^{9.10 .11}$ Furthermore the advances in the fabrication of mesoscopic and nanoscopic de- 
vices provide a great flexibility in designing systems for which one can measure and control processes dominated by AOC. One example would correspond to the situation where an electron tunnels through a system of two quantum dots joined via a small constriction. In that case the boundary conditions of the electrons on the far dot are changed once the tunneling electron enters the first dot, ${ }^{14,15}$ and as a consequence a change of one electron appears as a sudden perturbation to the remaining electrons. Another example is a mesoscopic tunnel junction containing a localized impurity level such that tunneling from the junction to the lead leaves behind a hole bound to the impurity. The resulting power-law singularities in the current-voltage characteristics were studied in Ref. 16 and observed in experiments with two- and three-dimensional electrodes ${ }^{17}$ Large optical singularities in one-dimensional electron gases formed in semiconductor quantum wires were observed in Ref. 18 and theoretically explained as Fermi-edge singularities 19

Aspects of AOC in disordered or mesoscopic systems have been addressed in various publications ${ }^{20,21.22 .23}$ AOC in disordered simple metals and its influence on $\mathrm{x}$-ray photoemission spectra (where the excited core electron leaves the metal and the edge behavior is determined by the AOC response alone) was studied by Chen and Kroha ${ }^{20}$ In Ref. 22, Vallejos and coworkers studied AOC in chaotic mesoscopic systems for a weak perturbation modeled by a random matrix. They found large fluctuations of the AOC overlap that is non-zero in general due to the incomplete orthogonality. Small overlaps are correlated with avoided level crossings between the highest filled and the lowest empty level. This motivated the analytic study of a two-level model to which we refer below. Mesoscopic conductors with diffusive disorder were studied with respect to AOC by Gefen et al $l^{23}$ The dependence of the average AOC-overlap on the number $M$ of electrons was found to be dimension dependent, and to show a stronger dependence on $M$ in two dimensions than in higher dimensions. In agreement with the results by Vallejos et al. and the results we present here, a broad distribution of overlaps is seen, with the small-overlap tail of the distribution being related to fewlevel statistics near the Fermi-energy. Another key result was that the disorder-averaged AOC overlap depends non-monotonically on the disorder. This becomes clear in the limit of strong disorder where the electrons are strongly localized and cannot be sensitive to the addition of another impurity, and was confirmed in their numerical results.

As already mentioned, here we will more specifically consider the case of a short range (rank one), not necessarily small, perturbation, ${ }^{3.4 .7 .21}$ which applies for the description of the perturbation created by the core hole. We will furthermore consider a generic chaotic mesoscopic system, such as a quantum dot or nanoparticle, for which a random matrix theory (RMT) model of the conduction electrons applies. This particular regime will show some similarities with both the diffusive systems studied in Ref. 23 and the non-rank-one perturbative regime investigated by Vallejos et al., $\stackrel{22}{,}$ but will also display interesting specific properties.

Beyond AOC, Fermi-edge singularities in the mesoscopic regime were considered in Refs. 8 , 15, and 16 . We will con- sider the full mesoscopic x-ray edge problem and the resulting FES in photoabsorption spectra of quantum dots or nanoparticles in the second paper of this series,$\underline{I I}$ and concentrate on $\mathrm{AOC}$ in the present, first paper.

The paper is organized as follows. In Sec. [1] we will introduce our model of a rank one perturbation on discrete (bulklike or chaotic) energy levels and apply it to the study of AOC. We investigate the properties of the ground state overlap probability distribution $P\left(|\Delta|^{2}\right)$ and show that the phase shift at the Fermi energy determines the overlap fluctuations. The presence of a bound state is addressed in Sec. IIII and its role for the symmetry of overlap distributions for positive and negative perturbations is discussed. Knowing that the origin of the fluctuations lies in the levels nearest to the Fermi energy allows us to derive an analytic formula for the overlap distribution in the limit of strong perturbations in Sec. IV We end this first paper with a summary on AOC in generic mesoscopic systems.

\section{ANDERSON ORTHOGONALITY CATASTROPHE FOR MESOSCOPIC CHAOTIC SYSTEMS}

Anderson orthogonality catastrophe refers to the vanishing of the overlap $\Delta$ between the system's many-body ground states before and after a finite range scattering potential is suddenly applied, and is complete only in the thermodynamic limit $\frac{12}{1 n}$ Inis Section we will discuss the effect of a finite number of particles in a mesoscopic system as well as the role of mesoscopic fluctuations. As the spin variable does not play a particular role here, we shall not take it into account (except for the discussion of the Friedel sum rule). We therefore describe the unperturbed system by the Hamiltonian

$$
\hat{H}_{0}=\sum_{k=0}^{N-1} \epsilon_{k} c_{k}^{\dagger} c_{k}
$$

where the operator $c_{k}^{\dagger}$ creates a particle in the unperturbed eigenstate $\varphi_{k}(\mathbf{r})$ corresponding to the eigenenergy $\epsilon_{k}$. We want to consider a situation where the perturbing potential is created by the hole left by a core electron excited into the conduction band, which due to screening by the other electron is a short range potential. We shall therefore use for the perturbation a rank one, contact, potential

$$
\hat{V}_{c}=v_{c} \Omega f^{\dagger}\left(\mathbf{r}_{c}\right) f\left(\mathbf{r}_{c}\right),
$$

$\left[f\left(\mathbf{r}_{c}\right)=\sum_{k} \varphi_{k}\left(\mathbf{r}_{c}\right) c_{k}\right]$, that only acts at the location $\mathbf{r}_{c}$ of the core hole ( $\Omega$ is the volume in which the electrons are confined, and the parameter $v_{c}$ defines the strength of the potential). Introducing $\tilde{c}_{\kappa}^{\dagger}$ as creator of a particle in the perturbed orbital $\psi_{\kappa}(\mathbf{r})$ (we will use Greek indices to indicate the perturbed system), we obtain the diagonal form of the perturbed Hamiltonian as

$$
\hat{H}=\hat{H}_{0}+\hat{V}_{c}=\sum_{\kappa} \lambda_{\kappa} \tilde{c}_{\kappa}^{\dagger} \tilde{c}_{\kappa} .
$$

We will refer to the many-body, Slater-determinant ground states of the unperturbed and the perturbed system as $\left|\Phi_{0}\right\rangle$ 
and $\left|\Psi_{0}\right\rangle$, respectively. Their overlap is $\Delta=\left\langle\Psi_{0} \mid \Phi_{0}\right\rangle$. In the ground state the $M$ conduction electrons fill the lowest of the $N$ levels, i.e. the levels $i=0 \ldots M-1$ [see also Fig. 7 (a)]. Whenever practical, we will use the index $i$ ( $\mu$ for perturbed levels) for levels below $E_{F}$ and $j(\gamma)$ for levels above $E_{F}$, and $k(\kappa)$ when reference to all levels is made.

\section{A. Bulk-like systems}

In the bulk the orbital momentum $l$ is a good quantum number, and, because the core hole potential (2) is rotationally invariant, only the $l=0$ sector couples to the perturbation. Within this sector, the unperturbed energy levels $\left\{\epsilon_{k}\right\}$ near the Fermi energy can be taken equidistantly spaced and, with a proper choice of the origin of the phase of the wavefunctions we can take $\phi_{k}\left(\mathbf{r}_{c}\right)=$ const. $=1 / \sqrt{\Omega}$.

In the following, we will call "bulklike" a model which, as the $l=0$ sector of a bulk system, has no fluctuations for the eigenlevel spacing nor for the amplitude of the wavefunction at the impurity. We shall, however, assume finite the number of levels within the bandwidth. In this subsection we shall review briefly some properties of this bulklike system. is

In the absence of mesoscopic fluctuations, the perturbation

$$
\hat{V}_{c}=v_{c} \sum_{k k^{\prime}} c_{k}^{\dagger} c_{k^{\prime}}
$$

Introducing the transformation matrix $\mathbf{a}=\left(a_{k \kappa}\right)$,

$$
\psi_{\kappa}=\sum_{k=0}^{N-1} a_{k \kappa} \varphi_{k},
$$

one can check easily that the perturbed eigenstates $\left\{\lambda_{\kappa}\right\}$ and the coefficients $a_{k \kappa}$ fulfill the familiar equations ${ }^{7}$

$$
\begin{aligned}
0 & =1-v_{c} \sum_{k=0}^{N-1} \frac{1}{\lambda_{\kappa}-\epsilon_{k}}, \\
a_{k \kappa} & =-\frac{\nu_{\kappa}}{\lambda_{\kappa}-\epsilon_{k}} \\
\frac{1}{\left|\nu_{\kappa}\right|^{2}} & =\sum_{k=0}^{N-1} \frac{1}{\left(\lambda_{\kappa}-\epsilon_{k}\right)^{2}} .
\end{aligned}
$$

Eq. (5) allows one to find the perturbed eigenvalues $\lambda_{\kappa}$ as a function of the unperturbed energies $\epsilon_{k}$ and the perturbation strength $v_{c}$. This is illustrated in Fig. 11 where the perturbed eigenvalues $\lambda_{\kappa}$ are found as intersection points of the function

$$
f(\lambda) \stackrel{\text { def }}{=} \sum_{k} \frac{1}{\lambda-\epsilon_{k}}
$$

with the horizontal line $1 / v_{c}$. We have assumed a negative perturbation $v_{c}<0$ to account for the attractive perturbation caused by the core hole potential.

The effect of the perturbation and the shift between perturbed and unperturbed levels is generally characterized by

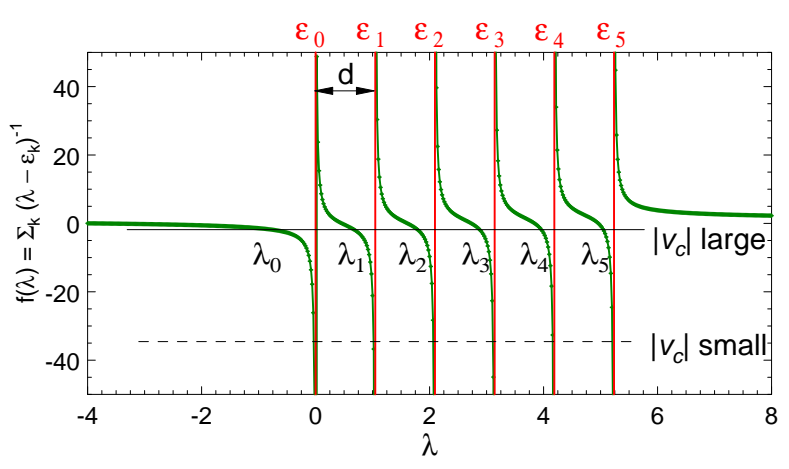

FIG. 1: (color online) Perturbation of a bulklike material with six initially equidistant energy levels $\epsilon_{k}\left(0 \leq \epsilon_{k} \leq 2 \pi\right.$, mean level spacing $d$ ) by a rank one perturbation of strength $v_{c}<0$. The perturbed eigenvalues $\lambda_{\kappa}$ arise from a graphical solution of Eq. (5) and are shifted to lower energies (attractive interaction). This shift increases as the perturbation becomes stronger; see the horizontal lines corresponding to two different potential strengths. The strongest perturbation shifts the perturbed levels right to the middle of two neighboring unperturbed levels $\epsilon_{k}, \epsilon_{k+1}$ (strictly, this applies in the center of the band - see the discussion of boundary effects).

the partial-wave phase shifts $\delta_{l}$ at the Fermi energy for the orbital channel $l$. The phase shifts obey the Friedel sum rule

$$
Z=\sum_{l} 2(2 l+1) \delta_{l} / \pi
$$

where the factor of two accounts for spin and $Z=-1$ in the case of photoabsorption. For a localized rank-one perturbation which, as mentioned above, is spherically symmetric, all $\delta_{l}$ with $l \neq 0$ are zero. In this case the Friedel sum rule Eq. 8. implies $\delta_{0}=-\pi / 2$.

When $E_{F}$ is in the middle of the conduction band, $\delta_{0}$ is related to the potential $v_{c}$ and the mean level spacing $d$ by ${ }^{21}$

$$
\delta_{0}=\arctan \frac{\pi v_{c}}{d} .
$$

$\delta_{0}=-\pi / 2$ therefore corresponds to a strong perturbation $\left|v_{c} / d\right| \gg 1$. We will therefore be mainly interested in AOC in this limit, although we shall also consider other cases.

For the finite number of particles that we consider in the following, boundary effects are important. First, the phase shift varies (monotonically) with the level number, and only for levels at the band center Eq. (9) holds. For levels off-center, the number of energy levels on the left and right is unbalanced and leads to additional pushing from the side with the surfeit. This can already be guessed from inspecting Fig. 1

This effect can easily be taken into account by noting that, for any perturbed level $\lambda_{\kappa}$ [assuming for instance $0 \leq \kappa \leq$ $(N-1) / 2$ ], Eq. (5) can we rewritten as

$$
\sum_{k=0}^{2 \kappa-1} \frac{1}{\lambda_{\kappa}-\epsilon_{k}}=\frac{1}{v_{\kappa}}
$$

where we have defined

$$
\frac{1}{v_{\kappa}} \stackrel{\text { def }}{=} \frac{1}{v_{c}}-\sum_{k=2 \kappa}^{N-1} \frac{1}{\epsilon_{k}-\lambda_{\kappa}} .
$$


Since $\kappa$ is now in the middle of the range $[0,2 \kappa-1]$, we see that Eq. (9) can be generalized to give a level-dependent phase shift $\delta_{\kappa}=\arctan \pi v_{\kappa} / d$, where evaluating the sum as an integral yields

$$
\begin{aligned}
& \frac{1}{v_{\kappa}}=\frac{1}{v_{c}}+\frac{1}{d} \ln \frac{N-\frac{1}{2}-\kappa}{\kappa+\frac{1}{2}} \text { if } \kappa \in\left(0, \frac{N-1}{2}\right), \\
& \frac{1}{v_{\kappa}}=\frac{1}{v_{c}}-\frac{1}{d} \ln \frac{N-\frac{1}{2}-\kappa^{\prime}}{\kappa^{\prime}+\frac{1}{2}} \text { if } \kappa \in\left(\frac{N-1}{2}, N-1\right) .
\end{aligned}
$$

Note that away from the band center, $\delta_{\kappa}$ is no longer confined to the interval $[-\pi / 2, \pi / 2]$ but can take values in the range $[-\pi, \pi]$.

A second boundary effect becomes important for strong perturbations $v_{c} / d \lesssim-1$ that induce a large shift of the lowest perturbed energy level towards $-\infty$ (see Fig. 1]. We will discuss the effect of such a bound state in detail in Sec. IIII

A remarkable property of rank one perturbations such as a contact potential is that the overlap between the many-body ground states $\left|\Phi_{0}\right\rangle$ and $\left|\Psi_{0}\right\rangle$ of $\hat{H}_{0}$ and $\hat{H}$ can be expressed as 7

$$
|\Delta|^{2}=\left|\left\langle\Psi_{0} \mid \Phi_{0}\right\rangle\right|^{2}=\prod_{i=0}^{M-1} \prod_{j=M}^{N-1} \frac{\left(\lambda_{j}-\epsilon_{i}\right)\left(\epsilon_{j}-\lambda_{i}\right)}{\left(\lambda_{j}-\lambda_{i}\right)\left(\epsilon_{j}-\epsilon_{i}\right)} .
$$

In other words, all the required information is contained in the eigenvalues $\{\epsilon\},\{\lambda\}$. In the bulklike situation we have considered so far, the $\{\lambda\}$ are uniquely determined from the $\{\epsilon\}$, yielding the bulklike overlap $\left|\Delta_{b}\right|^{2}$. Analytic formulas for $\left|\Delta_{b}\right|^{2}$ were discussed in Ref. 7. For half filling, $M=N / 2$, and assuming a level-independent phase shift, the relation

$$
\left|\Delta_{b}\right|^{2}=\left(\frac{N}{2}\right)^{-\frac{\delta_{0}^{2}}{\pi^{2}}} e^{-\frac{\delta_{0}^{2}}{\pi^{2}} \mathcal{F}}
$$

is easily derived. The factor $\mathcal{F} \approx \frac{1}{2} \sum_{k=1}^{\infty} \frac{1}{k^{2}} \approx 0.822$ is a correction to the exponent due to the discreteness of the levels.

\section{B. Mesoscopic systems}

We now consider a generic chaotic mesoscopic system. In that case, because of the confinement, both energies and wavefunctions will display mesoscopic fluctuations from level to level or as an external parameter is varied. $9,10,11$ This implies that the effective strength of the contact potential felt by electron $k$ cannot be assumed constant. More precisely, introducing $u_{k} \equiv \sqrt{\Omega} \varphi_{k}\left(\mathbf{r}_{c}\right)$, such that $\left\langle\left|u_{k}\right|^{2}\right\rangle=1$, we now have

$$
\hat{V}_{c}=v_{c} \sum_{k k^{\prime}} u_{k}^{*} u_{k^{\prime}} c_{k}^{\dagger} c_{k^{\prime}}
$$

Accordingly, the generalized version of Eqs. (5)-(7) for the perturbed energy levels $\lambda_{\kappa}$ reads

$$
0=1-v_{c} \sum_{k=0}^{N-1} \frac{\left|u_{k}\right|^{2}}{\lambda_{\kappa}-\epsilon_{k}}
$$

$$
\begin{aligned}
a_{k \kappa} & =-\frac{\nu_{\kappa} u_{k}}{\lambda_{\kappa}-\epsilon_{k}}, \\
\frac{1}{\left|\nu_{\kappa}\right|^{2}} & =\sum_{k=0}^{N-1} \frac{\left|u_{k}\right|^{2}}{\left(\lambda_{\kappa}-\epsilon_{k}\right)^{2}}, \\
u_{k}^{2} & =\frac{1}{v_{c}} \frac{\prod_{\kappa=0}^{N} \lambda_{\kappa}-\epsilon_{k}}{\prod_{\kappa \neq k}^{N} \epsilon_{\kappa}-\epsilon_{k}}, \\
\tilde{u}_{\kappa} & =\sum_{k=0}^{N-1} a_{k \kappa} u_{k} \equiv-\frac{\nu_{\kappa}}{v_{c}},
\end{aligned}
$$

and the bulklike situation is recovered by setting $u_{k}^{2}=$ $\left\langle\left|u_{k}\right|^{2}\right\rangle=1$. Note that we can choose the phase in the wavefunctions $\varphi_{k}$ and $\psi_{\kappa}$ such that their value at $\mathbf{r}_{c}$ is real. It is then evident that $u_{k}, a_{k \kappa}$, and $\tilde{u}_{\kappa}$ are also real.

For a chaotic system, a good model for the eigenvalues and eigenvector fluctuations is given by the classic ensembles of Random Matrix Theory (RMT) ${ }^{24,25}$ Most commonly the Gaussian orthogonal (unitary) ensembles, GOE (GUE), are used to describe the energy eigenvalues of chaotic systems in the presence (absence) of time reversal symmetry. Here however, because we would like to model systems with constant mean density of states, we will rather consider the corresponding Circular Ensembles COE and $\mathrm{CUE}^{25}$ which display the same fluctuations but have by construction a mean density of eigenphases $\exp \left(i \epsilon_{k}\right)$ that is uniformly distributed on the unit circle. Since time reversal symmetry can easily be broken by applying a magnetic field, we will address both ensembles.

The fluctuations in the $\{\epsilon\}$ and $\{\lambda\}$ are not independent. Under the hypothesis that the unperturbed eigenenergies are distributed according to the circular ensembles, and accordingly that the eigenvectors follow a Porter-Thomas distribution, the joint probability distribution $P(\{\epsilon\},\{\lambda\})$ was shown by Aleiner and Matveev ${ }^{21}$ to be

$$
P(\{\epsilon\},\{\lambda\}) \propto \frac{\prod_{i>j}\left(\epsilon_{i}-\epsilon_{j}\right)\left(\lambda_{i}-\lambda_{j}\right)}{\prod_{i, j}\left|\epsilon_{i}-\lambda_{j}\right|^{1-\beta / 2}} e^{-\frac{\beta}{2} \sum_{i}\left(\lambda_{i}-\epsilon_{i}\right) / v_{i}}
$$

with the constraint $\epsilon_{i-1} \leq \lambda_{i} \leq \epsilon_{i}$ and $\beta=1$ in the COE case whereas $\beta=2$ for CUE. In the middle of the band $v_{i}=v_{c}$, away from the band center, boundary effects have to be taken into account according to Eq. (12). It is interesting that a joint probability distribution can also be found in the more general, non-rank-one $\operatorname{case}^{26}$ that we do not consider here.

The ground state overlap $\Delta$ is expressed entirely in terms of the unperturbed and perturbed eigenvalues $\{\epsilon\}$ and $\{\lambda\}$ [see Eq. (13)]. Therefore, the joint distribution (20) contains all the information required to build the overlap distribution $P\left(|\Delta|^{2}\right)$.

In Section IV we shall see that a slightly different route is actually more effective to obtain analytic expressions for $P\left(|\Delta|^{2}\right)$. However, the present approach is perfectly adapted to constructing numerically this distribution for any value of the parameters $M, N, v_{c}$, and $\beta$. Indeed, we can use Eq. 20. as the basis of a simple Metropolis algorithm to generate ensembles of $(\{\epsilon\},\{\lambda\})$ with the proper joint probability distribution. Applying Eq. (13), we then build the distribution of 

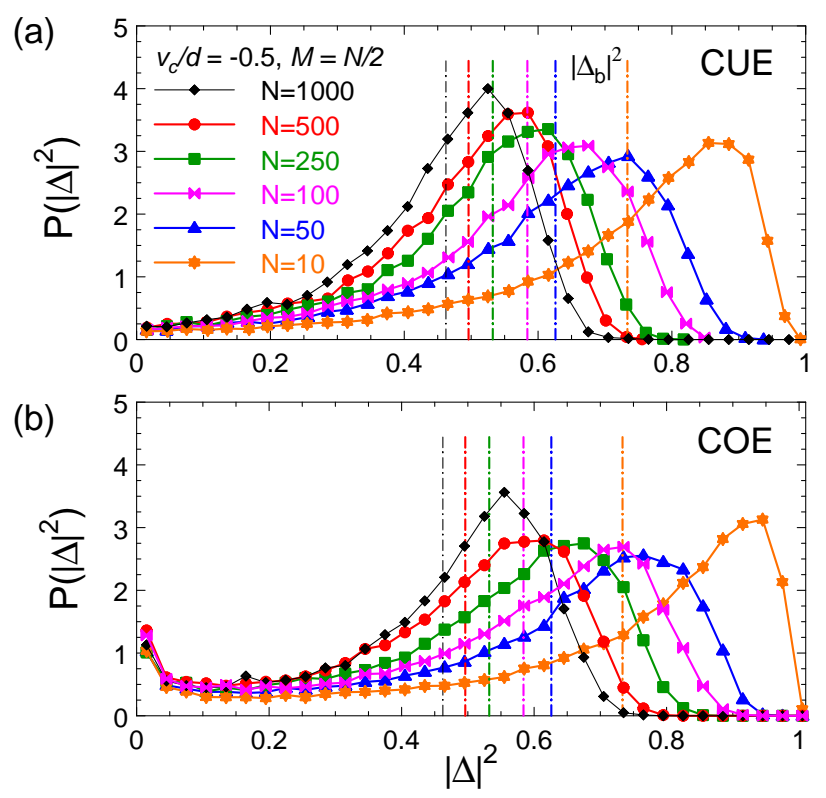

FIG. 2: (color online) Distribution of ground-state overlaps in a mesoscopic system as the number of particles is increased at halffilling, $M=N / 2$, for the (a) CUE and (b) COE cases. The perturbation strength is chosen intermediate, $v_{c} / d=-0.5$. The dashdotted lines indicate the bulklike overlaps $\left|\Delta_{b}\right|^{2}$. The curves illustrate the power-law characteristic of AOC that makes it a truly thermodynamic-limit effect: even with $M=1000$ electrons in the system, the most probable overlap $|\Delta|^{2}$ is as big as 0.46 , indicating that a bulk-based discussion of AOC in mesoscopic systems can be inaccurate. Note that for this intermediate perturbation strength the overlap distribution exhibits a clear maximum and a difference in CUE vs. COE for small overlaps. (In order to make the statistical error essentially negligible, 20000 realizations of the random matrix were used in producing each curve.)

ground state overlaps. Examples for overlap distributions obtained in this way are shown in Fig. 2 for systems with and without time-reversal symmetry and an intermediate perturbation strength. The evolution of AOC as the number of particles is increased is clearly visible as a shift of the probability density to smaller values of $|\Delta|^{2}$. However, since AOC is a power-law effect even $N=1000$ is far away from the thermodynamic limit, cf. Eq. (14) that can also be used as a rough estimate for the mean overlap in the mesoscopic case. We point out the different behavior for very small overlaps in the CUE vs. COE case. It originates in the difference of the Porter-Thomas distributions and will be discussed in Sec.IV

In the metallic $\mathrm{x}$-ray edge problem all relevant properties are known to depend only on the phase shift at the Fermi energy, and a natural question is whether this remains true in the mesoscopic case for the groundstate overlap. In Fig. 3 (a) we therefore compare overlap distributions for different parameter sets $\left\{N, M, v_{c} / d\right\}$ all of which yield a phase shift $\delta_{F} \approx \arctan \left(\pi v_{i=M} / d\right) \sim-\pi / 2$ at the Fermi energy $E_{F}$. The curves differ, as do the corresponding bulklike overlaps $\left|\Delta_{b}\right|^{2}$ indicated by the vertical lines. However, after scaling by the bulklike overlaps all curves coincide, Fig. 3 b). There-
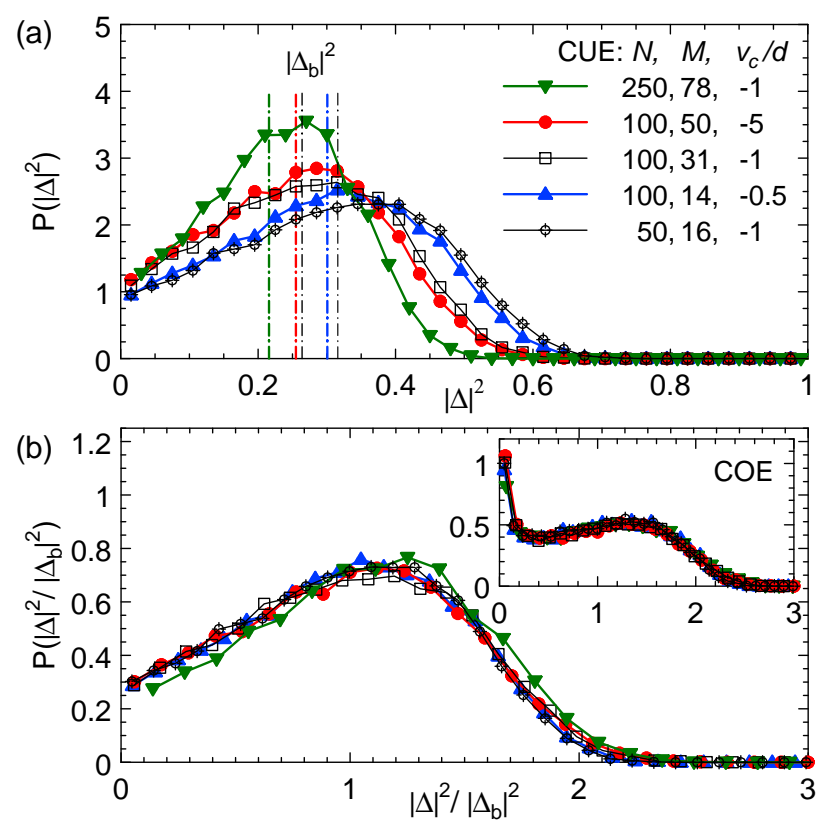

FIG. 3: (color online) Ground state overlap in the absence of timereversal symmetry (CUE case) for different parameter realizations corresponding to a phase shift of approximately $-\pi / 2$ at the Fermi energy. (a) Before, (b) after scaling with the bulklike result $\left|\Delta_{b}\right|^{2}$ (dash-dotted lines, $\left|\Delta_{b}\right|^{2}$ increases with the legend entries). The scaled result for COE is given in the inset of (b), see Ref. 8 for the unscaled result in that case.

fore, whereas the individual $P\left(|\Delta|^{2}\right)$ depend independently on $N, M$, and $v_{c} / d$, the fluctuations of the overlap depend on the value of $\delta_{F}$ alone.

This fact allows one to provide the overlap distribution as a universal curve for a given phase shift $\delta_{F}$. In Figs. 4(a) and [5] a) results are shown for $N=100$ levels subject to a strong perturbation. The phase shift $\delta_{F}$ is varied by increasing the filling of the band from 2 to 98 electrons. The distributions are not scaled by the bulk overlap in order to allow easier comparison of the curves when they all range from 0 to 1 .

\section{Fluctuations of levels beyond the Thouless energy}

Strictly speaking a random matrix model applies only to chaotic energy levels on a scale of the Thouless energy $E_{\mathrm{Th}}{ }^{10.11 .27 .28}$ In the semiclassical limit $E_{\mathrm{Th}}$ is much larger than the mean level spacing, but significantly smaller than the Fermi energy $E_{F}$. Therefore one may question the model we use where the full bandwidth is described by RMT. It turns out, however, that the overlap distributions depend only on the fluctuation of the levels near the Fermi energy. In order to demonstrate this, we introduce a range-n approximation in which only $n$ of the levels on either side of the Fermi energy are left free to fluctuate, while all the others are kept fixed at their mean position. To gauge the accuracy of this approximation, we perform a Kolmogorov-Smirnov test ${ }^{29}$ In Fig. 6 the result of this test, namely the maximum deviation of the range- 

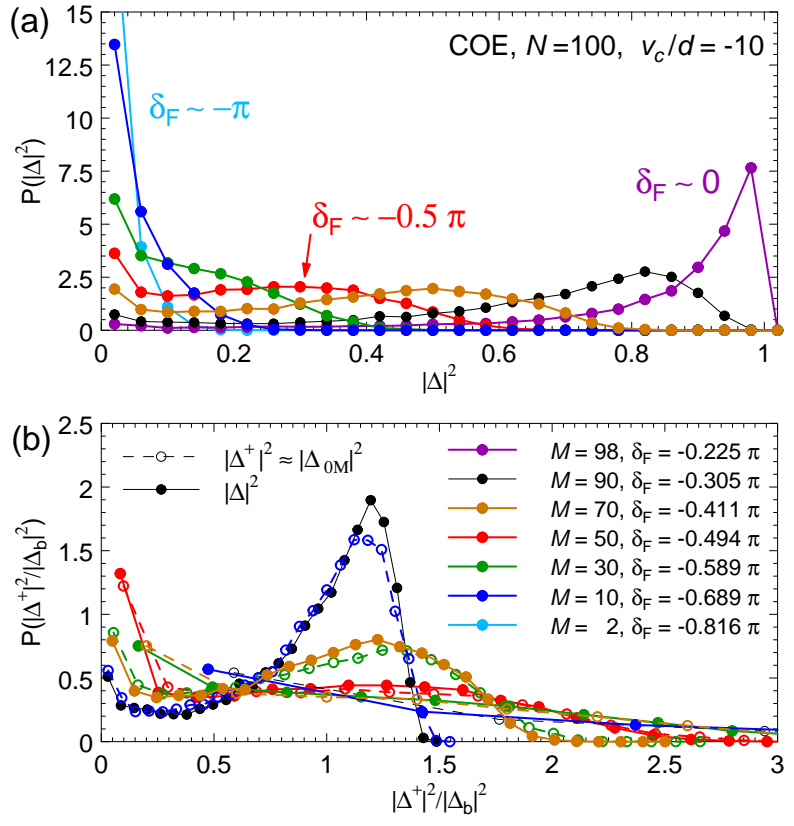

FIG. 4: (color online) (a) Probability distribution $P\left(|\Delta|^{2}\right)$ for the ground state overlap (COE, $N=100, v_{c} / d=-10$ ). The number $M$ of electrons is varied, corresponding to a varying phase shift $\delta_{F}$ at the Fermi energy. The curves (here not scaled by the bulklike overlaps) illustrate the characteristics of $P\left(|\Delta|^{2}\right)$ for a given phase shift. For comparison, recall that at constant filling, a decreasing phase shift corresponds to increasing perturbation strength. (b) Comparison of the bulk-scaled probability distributions of the ground state overlaps for a negative (solid line, $|\Delta|^{2}$ ) and positive (dashed line) perturbation. The overlap $\left|\Delta^{+}\right|^{2}$ is, up to the negligible influence of the run-away states, equivalent to the overlap $\left|\Delta_{0 M}\right|^{2}$ shown here as dashed line. It will be of importance in the discussions of the $\mathrm{x}$-ray absorption part. The pairing of curves whose phase shifts moduli add up to $\pi$ is nicely illustrated in this graph.

$n$ cumulative distribution from the exact cumulative distribution of the overlap, is shown. Clearly, the deviation decreases to almost zero as soon as the fluctuations of a few levels near $E_{F}$ are included. The convergence with $n$ appears furthermore faster for the larger perturbation strengths in which we are interested for the $\mathrm{x}$-ray edge problem. This retrospectively justifies the use of a full random matrix to model the statistical properties of our chaotic systems, as the levels beyond the Thouless energy for which such a description does not hold do not affect the overlap distribution.

\section{BOUND STATES AND SYMMETRY BETWEEN POSITIVE VS. NEGATIVE PERTURBATION}

As soon as the perturbation strength becomes significant, i.e. for attractive perturbations $v_{c} / d \lesssim-1$, the shift of the lowest perturbed level, $\lambda_{0}-\epsilon_{0}$, starts to become large. Analogously, for positive perturbation strengths larger than $v_{c} / d \gtrsim 1$, the same boundary effect now shifts the highest perturbed level $\lambda_{N-1}$ to very high energies, see Figs. 11 and 7
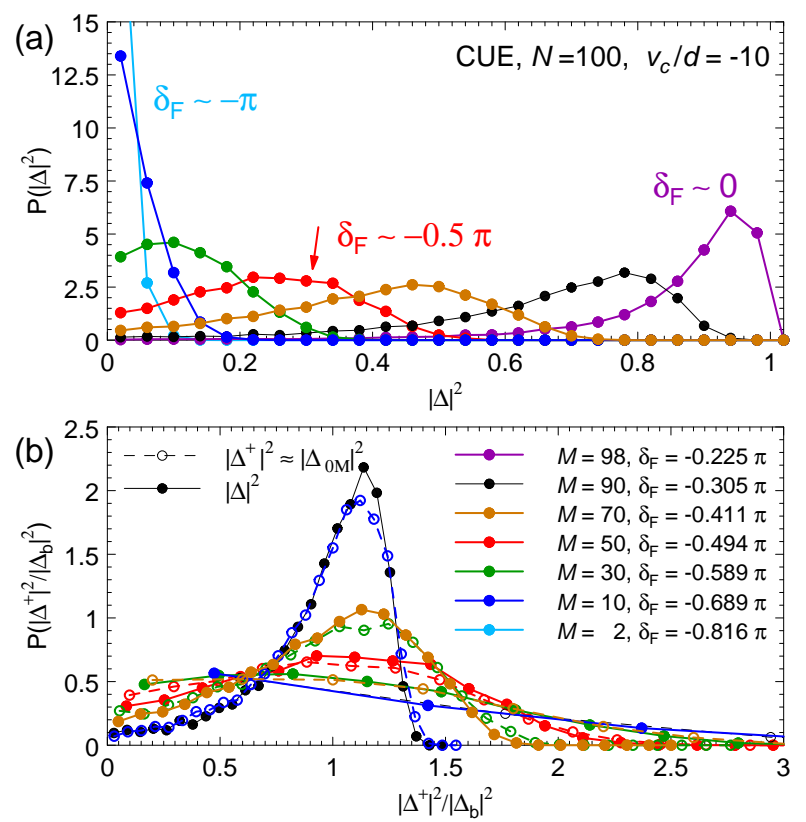

FIG. 5: (color online) Same as in Fig. 4 but for CUE. Note the difference in the distributions for small overlaps $|\Delta|^{2}$.

These so-called run-away levels correspond to the formation of a bound state $e^{7.30,31.32}$ which entirely screens the impurity. They can be found, respectively, at

$$
\begin{aligned}
\lambda_{0}-\epsilon_{0} & =-\frac{N d}{e^{d /\left|v_{c}\right|}-1} \quad\left(v_{c}<0\right), \\
\lambda_{N-1}-\epsilon_{N-1} & =+\frac{N d}{1-e^{-d / v_{c}}} \quad\left(v_{c}>0\right) .
\end{aligned}
$$

The fluctuations of these levels will be neglected, ${ }^{33}$ since in the limit of very strong perturbations, $\left|v_{c}\right| / d \gg 1$, the runaway level does not affect the value for the overlap, Eq. (13), because the terms involving $\lambda_{0}$ (or $\lambda_{N-1}$, respectively) yield ratios of one. Therefore, one has only to consider the $N$ unperturbed levels $\{\epsilon\}$ and the $N-1$ perturbed levels $\lambda_{\kappa}$ "in between". In this case of very strong perturbations, the perturbed levels are maximally shifted: in the center of the band, the $\lambda_{\kappa}$ lie, on average, exactly in the middle between the two neighboring unperturbed levels $\epsilon_{\kappa-1}$ and $\epsilon_{\kappa}$ (or $\epsilon_{\kappa}$ and $\epsilon_{\kappa+1}$ ). Therefore, a strong negative perturbation $v_{c}$ (i.e. $\left.v_{c} / d \ll-1, \quad \delta_{F}=-\pi / 2\right)$ and a strong positive perturbation $v_{c}^{+}$(i.e. $v_{c}^{+} / d \gg 1, \delta_{F}^{+}=\pi / 2$ ) are indistinguishable, see Fig. 7 This means in particular that the corresponding overlap distributions $P\left(|\Delta|^{2}\right)$ and $P\left(\left|\Delta^{+}\right|^{2}\right)$ are identical.

In the case of intermediate perturbation strengths $v_{c} / d \sim$ \pm 1 , a level shift $\delta_{F}$ associated with the negative perturbation corresponds to a phase shift $\delta_{F}^{+}=\pi-\left|\delta_{F}\right|$ when the level scheme is interpreted as originating from some positive perturbation $v_{c}^{+}\left(\neq\left|v_{c}\right|\right.$ in general), see Fig. [7 $\mathrm{c}$ ). We therefore expect again the two corresponding bulk-scaled overlap dis- 


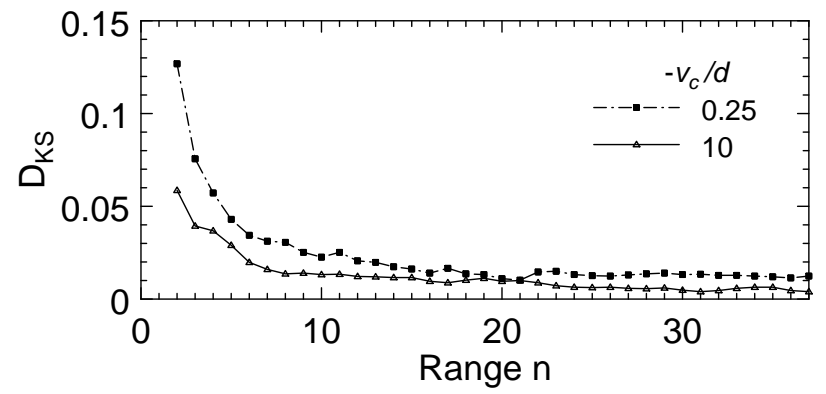

FIG. 6: Accuracy of the range- $n$ approximation using the Kolmogorov-Smirnov test (COE, $N=100, M=50$ ). The maximum deviation of the range- $n$ cumulative distribution from the exact cumulative distribution of the overlap is shown as a function of $n$. The rapid approach to 0 , especially in the strong perturbation case, shows that fluctuation of only those levels near $E_{F}$ is important.

tributions to be identical, and the (symmetry) relation

$$
P\left(\frac{|\Delta|^{2}}{\left|\Delta_{b}\right|^{2}}\right)_{v_{c}, \delta_{F}}=P\left(\frac{\left|\Delta^{+}\right|^{2}}{\left|\Delta_{b}^{+}\right|^{2}}\right)_{v_{c}^{+}, \delta_{F}^{+}=\pi-\left|\delta_{F}\right|}
$$

to hold.

In Figs. 4 and 5 overlap distributions for phase shifts $0>$ $\delta_{F}>-\pi$ are shown. Starting with an almost full band ( $M=98$ particles on $N=100$ levels) and a small negative phase shift, $\delta_{F}$ is lowered (the effective potential strength raised) as the filling is decreased, passing $\delta_{F} \approx-\pi / 2$ at half filling. The corresponding overlap distributions are shown in Fig. 4 for the COE situation and in Fig. 5 a for a CUE case. In the lower panels (b) of Figs. 4 and 5 overlap distributions belonging to a negative phase shift $\delta_{F}\left(\right.$ and $\left.v_{c}<0\right)$ and the associated positive phase shift $\delta_{F}^{+}=\pi-\left|\delta_{F}\right|$ (and $v_{c}^{+}>0$ ) are compared and seen to (almost) coincide pairwise after scaling by the respective bulk overlaps (the small deviations are due to the finite number of discrete levels available). Note that the bulk overlaps $\left|\Delta_{b}^{+}\right|^{2}$ are very similar in each pair, but rather different for different pairs, thereby causing the stretching of the $\mathrm{x}$-axis.

In the context of the $\mathrm{x}$-ray edge problem (strong negative perturbation), a situation that is equivalent to a positive perturbation becomes of importance when the core electron is excited to the level $\lambda_{M}$ just above the Fermi energy $E_{F}$. The consequences and more details will be discussed in II.

\section{ANALYTIC RESULTS FOR THE OVERLAP DISTRIBUTION}

We finish with the derivation of analytic results for the overlap distribution. We argued in Sec. II that the fluctuations in the ground state overlap are dominated by fluctuations of the energy levels around the Fermi energy (see again Fig. 6). We now want to deepen this discussion and investigate to what extent an analytic understanding of overlap probability distributions $P\left(|\Delta|^{2}\right)$ is possible. In particular we want to un- (a) $v_{c}<<-1$

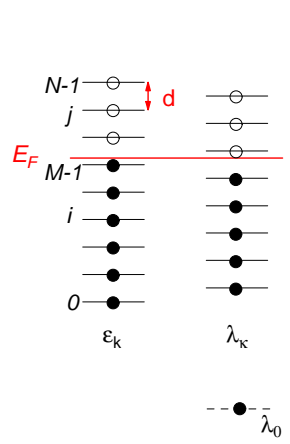

(b) $v_{c}>1$

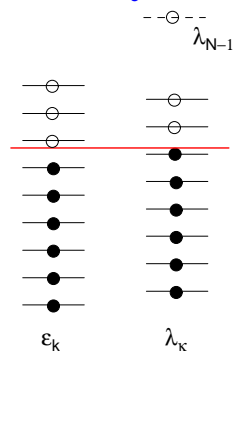

(c) $\left|v_{c}\right| \sim 1$

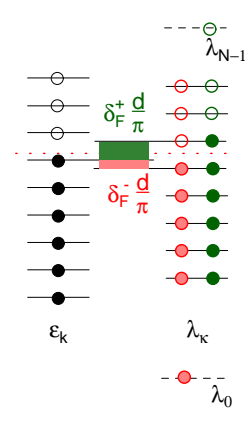

FIG. 7: (color online) Run-away level for strong perturbation which is (a) positive or (b) negative. For simplicity, the bulklike situation is shown. In the case of intermediate perturbations (c), the perturbed levels $\lambda_{\kappa}$ are not shifted to the center of the unperturbed level interval $\epsilon_{k}$. The phase shifts $\delta_{F}^{-}$(negative perturbation) and $\delta_{F}^{+}$(positive perturbation) are related by $\left|\delta_{F}^{-}\right|+\delta_{F}^{+}=\pi$. In the case of strong negative perturbations, as in the x-ray problem, $\lambda_{0}$ forms a bound state that screens the perturbation.

derstand two characteristic qualitative features of the overlap distribution (see for example Fig. 3): firstly $P\left(|\Delta|^{2}=0\right.$ ) is finite and characteristically different for the COE vs. CUE situation, and secondly $P\left(|\Delta|^{2}=1\right)$ is zero, i.e. $P\left(|\Delta|^{2}\right)$ drops to zero before the natural bound $|\Delta|^{2}=1$ is reached.

To begin, remember that in Eq. (13) for the overlap $|\Delta|^{2}$, all energy differences are taken between empty $(j \geq M)$ and occupied ( $i \leq M-1)$ levels. The smallest differences (of the order of the mean level spacing $d$ ) contain the energy levels $\epsilon_{M}, \epsilon_{M-1}$, and $\lambda_{M}$. We expect therefore that the small $\Delta$ part of the overlap distribution will be mainly given by the fluctuation of these terms.

To gain an analytic understanding of the overlap distribution, we therefore consider only the level closest to the Fermi energy as fluctuating whereas all other levels are assumed at their bulklike values (range-1 approximation). We therefore want to evaluate fluctuations of $\left(\lambda_{M}-\epsilon_{M-1}\right) /\left(\epsilon_{M}-\epsilon_{M-1}\right)$. For simplicity, we consider the half-filled case. Note that a similar two-level problem was studied by Vallejos et al. in Ref. 22, motivated by their observation that very small AOC overlaps were related to avoided level-crossings at the Fermi energy. In our present model, Eq. 15 for the rank one perturbation determines the position of the perturbed levels as solutions of the equation

$$
f(\lambda) \stackrel{\text { def }}{=} d \sum_{k} \frac{A_{k}}{\lambda-\epsilon_{k}} \equiv \frac{d}{v_{c}}
$$

where $A_{k}=\left|u_{k}\right|^{2}$ are the Porter-Thomas distributed wavefunction intensities,

$$
P(A)=\left\{\begin{array}{l}
e^{-A} \quad \text { for CUE } \\
\frac{1}{\sqrt{2 \pi A}} e^{-A / 2} \text { for } \mathrm{COE}
\end{array}\right.
$$

$\left[\langle A\rangle=1\right.$ and $\langle A\rangle^{2}=2(3)$ for CUE (COE) $]$. We will take only the fluctuations of the amplitudes $A_{M-1}$ and $A_{M}$ for 
the wavefunctions $\phi_{M-1}$ and $\phi_{M}$ explicitly into account. Accordingly, we separate the first two terms of the sum and comprise the remaining terms in a function $\delta f$ that is assumed to be a Gaussian random variable and contains the joint effect of all other levels and intensities,

$$
f(\lambda)=\frac{d A_{M-1}}{\lambda-\epsilon_{M-1}}+\frac{d A_{M}}{\lambda-\epsilon_{M}}+\delta f \equiv \frac{d}{v_{c}} .
$$

Equation (24) makes it possible already to understand at a qualitative level the finite probability for having $|\Delta|^{2}=0$ which corresponds to having $\lambda_{M}-\epsilon_{M-1}=0$. However, setting $\lambda=\lambda_{M}$ in Eq. (24) we see that the ratio $A_{M-1} /\left(\lambda_{M}-\epsilon_{M-1}\right)$ has to be finite. We conclude that having $\lambda_{M}-\epsilon_{M-1}=0$ is related to having $A_{M-1}=0$. From Eq. 23. we find $P(A=0)=1$ [or $\infty$ ] in the CUE [COE] case, thereby explaining both $P\left(|\Delta|^{2}=0\right)>0$ and the peak at $|\Delta|^{2}=0$ in the COE case.

To obtain a more quantitative description, we investigate $\delta f$ more closely. According to the assumptions of the range1 approximation, we replace the energy levels in $\delta f$ by their bulklike values. We furthermore neglect boundary effects that would make the phase shift dependent on the level number which is a good approximation for $N \rightarrow \infty$ at half-filling. Fixing the energy origin in the middle of the mean position of $\epsilon_{M-1}$ and $\epsilon_{M}$, we express $\delta f$ in the symmetric form

$$
\delta f=\sum_{k=1}^{\infty}\left(\frac{A_{k+M}}{\frac{\lambda}{d}-\left(k+\frac{1}{2}\right)}+\frac{A_{-(k+1)+M}}{\frac{\lambda}{d}+\left(k+\frac{1}{2}\right)}\right) .
$$

Fluctuations in $\delta f$ are due to the fluctuations in the intensities $A_{ \pm k}$ that follow the Porter-Thomas distributions given in Eq. (23). Expanding the mean value $\langle\delta f\rangle$ about the center of the band, $\lambda=0$, yields in lowest order $\langle\delta f\rangle=-2 \lambda S_{1}$ with

$$
S_{1}=\sum_{k=1}^{\infty} \frac{1}{\left(k+\frac{1}{2}\right)^{2}} \approx 0.9348
$$

Comprising all the randomness of the amplitudes $A_{k \neq M-1, M}$ in one random Gaussian variable $\xi$ with $P(\xi)=e^{-\xi^{2} / 2} / \sqrt{2 \pi}$, and writing explicitly the scale var $\delta f$ of the fluctuations around the mean value, we arrive at the approximation

$$
\delta f=\langle\delta f\rangle+\sqrt{\operatorname{var} \delta f} \xi=-2 \lambda S_{1}+\sqrt{\operatorname{var} \delta f} \xi
$$

with

$$
\operatorname{var} \delta f= \begin{cases}2 S_{1} & \text { for CUE } \\ 4 S_{1} & \text { for COE. }\end{cases}
$$

Setting $\epsilon_{M}-\epsilon_{M-1}=s$, the probability distribution $P(\lambda \mid s)$ is obtained by integrating over the distributions of $A_{M-1}, A_{M}$ and $\xi$. These integrals can only partially be solved analytically and, leaving out the further details of the calculation, the result for the CUE case $\left(\mu=\alpha_{M}-\alpha_{M-1}, \alpha_{0}=A_{M-1} /(\lambda+\right.$ $\left.s / 2), \alpha_{1}=-A_{M} /(\lambda-s / 2)\right)$ is

$$
P(\lambda \mid s)=\frac{1}{2 s \sqrt{\pi S_{1}}} \int_{-\infty}^{\infty} d \mu\left\{\exp \left[\mu \lambda-|\mu| \frac{s}{2}\right] \exp \left[-\frac{1}{4 S_{1}}\left(2 S_{1} \lambda+\frac{1}{v_{c}}+\mu\right)^{2}\right]\left[2 S_{1}\left(\frac{s^{2}}{4}-\lambda^{2}\right)+\mu \lambda+|\mu| \frac{s}{2}+1\right]\right\}
$$

and for the COE case

$$
\begin{aligned}
P(\lambda \mid s)=\frac{1}{\sqrt{(2 \pi)^{3} 4 S_{1}\left(\frac{s^{2}}{4}-\lambda^{2}\right)}} & \quad \int_{-\infty}^{\infty} d \mu\left[\exp \left[-\frac{1}{8 S_{1}}\left(2 S_{1} \lambda+\frac{1}{v_{c}}+\mu\right)^{2}+\mu \frac{\lambda}{2}\right]\right. \\
& \left.\times\left\{\left[2 S_{1}\left(\frac{s^{2}}{4}-\lambda^{2}\right)+\mu \lambda\right] K_{0}\left(\left|\frac{s \mu}{4}\right|\right)+2\left|\frac{s \mu}{4}\right| K_{1}\left(\left|\frac{s \mu}{4}\right|\right)\right\}\right],
\end{aligned}
$$

with $K_{0}, K_{1}$ the modified Bessel functions.

The remaining integral over $\mu$ can be performed in the CUE case using the (inverse) error function. Splitting the $\mu$-integration into integration from 0 to $\infty$ and from $-\infty$ to 0 , yielding results $P_{+}(\lambda \mid s)$ and $P_{-}(\lambda \mid s)$, and using the substitutions $A=$ $2 S_{1} \lambda+1 / v_{c}, B=2 S_{1}\left(s^{2} / 4-\lambda^{2}\right), a=1 /\left(4 S_{1}\right)$, and $b_{ \pm}=\left[s \pm 1 /\left(S_{1} v_{c}\right)\right] / 2$ we find

$$
P_{ \pm}(\lambda \mid s)=\frac{\exp \left(-A^{2} a\right)}{2 a C}\left\{ \pm\left(\lambda \pm \frac{s}{2}\right)+\sqrt{\frac{\pi}{a}} \exp \left(\frac{b_{ \pm}^{2}}{4 a}\right) \operatorname{erfc}\left(\frac{b_{ \pm}}{2 \sqrt{a}}\right)\left[B a \mp \frac{b_{ \pm}}{2}\left(\lambda \pm \frac{s}{2}\right)\right]\right\},
$$

and their sum yields $P(\lambda \mid s)=P_{+}(\lambda \mid s)+P_{-}(\lambda \mid s)$.

This expression simplifies considerably in the limit of very strong perturbations, $\left|v_{c}\right| \gg 1$. Then, the result becomes independent of $v_{c}$ (in particular independent of the sign - very strong attractive and repulsive interactions are equivalent), and takes the form

$$
P_{\infty}(\lambda \mid s)=\frac{\exp \left(-\lambda^{2} S_{1}\right)}{s \sqrt{\pi}}\left[s \sqrt{S_{1}}+\sqrt{\pi}\left(1-2 \lambda^{2} S_{1}\right) \exp \left(\frac{s^{2} S_{1}}{4}\right) \operatorname{erfc}\left(\frac{s \sqrt{S_{1}}}{2}\right)\right] .
$$

Clearly, this probability distribution is symmetric with respect $\quad$ to $\lambda=0$ as it should be. 
The contribution to the overlap, Eq. (13), that contains the energy levels closest to the Fermi energy is represented by $\left(\lambda_{M}-\epsilon_{M-1}\right) /\left(\epsilon_{M}-\epsilon_{M-1}\right) \equiv(\lambda+s / 2) / s$ in our present notation. To obtain the probability distribution $P^{F 1}(\lambda)$ of this quantity, we have to take into account the distribution of nearest neighbor spacings $s=\epsilon_{M}-\epsilon_{M-1}$ according to the Wigner surmise,

$$
P(s)= \begin{cases}\frac{32}{\pi^{2}} s^{2} \exp \left(-\frac{4}{\pi} s^{2}\right) & \text { for CUE } \\ \frac{\pi}{2} s \exp \left(-\frac{\pi}{4} s^{2}\right) & \text { for COE }\end{cases}
$$

The final result then reads

$$
P^{F 1}(\lambda)=\int_{0}^{\infty} d s s P(\lambda \mid s) P(s),
$$

This integrated result is however essentially indistinguishable from $P(\lambda \mid s=1)$.

The probability distribution of the range- 1 approximation, $P^{r 1}\left(\left|\Delta^{r 1}\right|^{2}\right)$, is straightforwardly obtained from $P^{F 1}(\lambda)$ by scaling with the bulklike quantity $\left|\Delta_{b}\right|^{2} /\left(1-\delta_{F} / \pi\right)$ [cf. Eq. (35) below] and shifting the argument by $1 / 2$. The resulting curves are presented in Fig. 8 for weak, intermediate, and strong perturbations. This analytic range- 1 result is compared to the full overlap distribution $P\left(|\Delta|^{2}\right)$ as well as the exact range- 1 and range- 2 approximations ${ }^{34}$ (CUE case, see Ref. 8 for the corresponding COE figure). The agreement between the analytic approximation and the exact range- 1 results is excellent. The analytic range- 1 result provides in particular a very good estimate for the full overlap distribution at small overlaps, independent of the perturbation strength. Moreover, in the limit of strong perturbations in which we are especially interested for the $\mathrm{x}$-ray edge problem, it provides a reasonable estimate of the full probability distribution $P\left(|\Delta|^{2}\right)$. The increase in the degree of symmetry of the range1 result (with respect to the maximum of the curve) as the perturbation strength is increased is clearly visible and confirmed by Eq. (32).

We would like to mention that Vallejos and coworkers in Ref. 22, and, based on their work, Gefen and coworkers in Ref. 23, found similar results, in particular that the distribution of small-overlaps is well represented by a two-level analytic model. However, there are few differences. For example, the difference between the (Gaussian) orthogonal and unitary case was less pronounced in their models, especially the increase of the probability $P\left(|\Delta|^{2}\right)$ towards $|\Delta|^{2} \rightarrow 0$, characteristic of our model, was not seen. Further, simple analytic expressions similar to the expressions Eqs. (29)-(30) could only be found for the orthogonal situation in Ref. 22 .

Eventually, we briefly remark on having $P\left(|\Delta|^{2}=1\right)=0$ for finite perturbation strength. Within a range-1-like approximation scheme, Eq. 13) can be written as

$$
\left|\Delta^{r 1}\right|^{2}=\frac{\left(\lambda_{M}-\epsilon_{M-1}\right)}{\left(\lambda_{M}-\lambda_{M-1}\right)} \frac{d}{d\left(1-\frac{\delta_{F}}{\pi}\right)}\left|\Delta_{b}\right|^{2},
$$

where we have normalized all differences by their bulklike values. Only the first term is fluctuating, between 0 and 1 , whereas the last two terms are numbers that are strictly
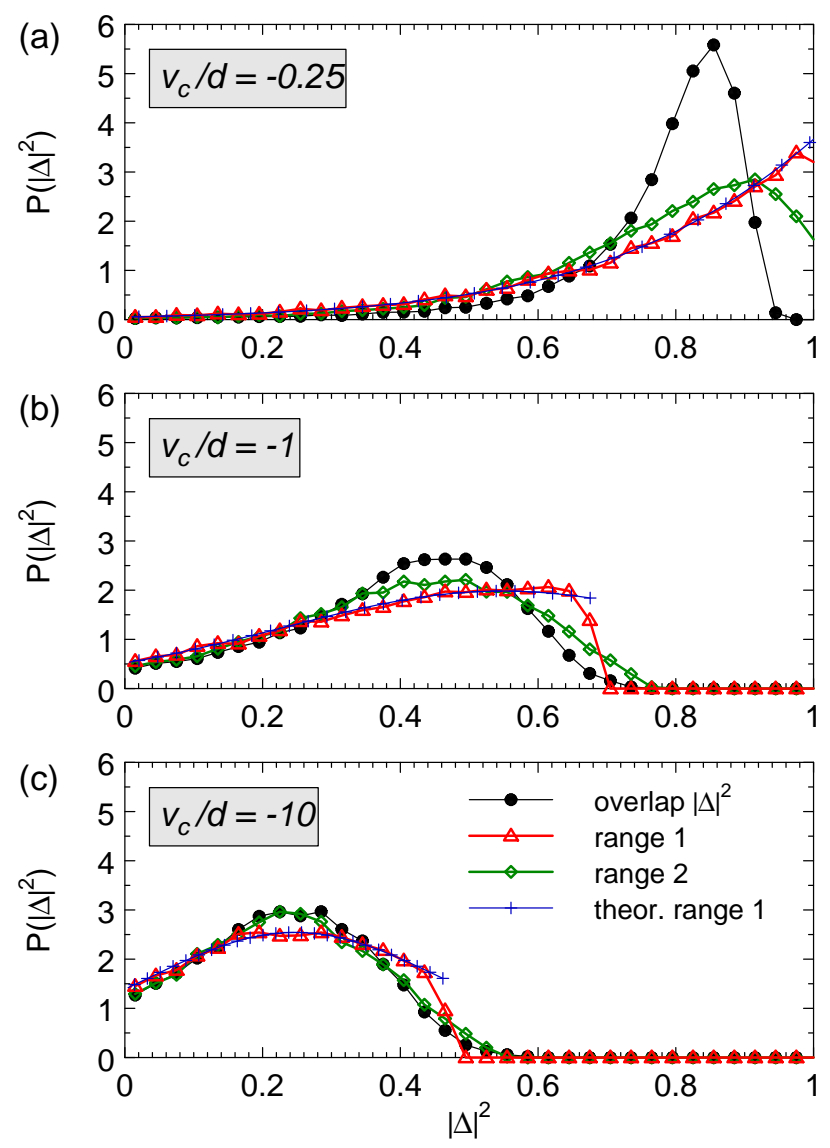

FIG. 8: (color online) Probability distributions $P\left(|\Delta|^{2}\right)$ for the ground state overlap ( $N=100, M=50$, CUE) for increasing potential strength: (a) weak $\left(v_{c} / d=-0.25\right)$, (b) intermediate $\left(v_{c} / d=-1\right)$, and (c) strong $\left(v_{c} / d=-10\right)$ perturbation. Shown are the exact distribution (dots) as well as the range-1 (triangles) and range-2 (diamonds) approximations. Clearly, the fluctuations come from the levels closest to the Fermi energy, and the range- 1 approximation provides a good estimate already for intermediate perturbation strengths as in (b). The crosses denote the analytic result for the range- 1 approximation, showing excellent agreement with the numerical results.

smaller than 1 and, furthermore, get smaller as the perturbation gets stronger. Accordingly, the upper boundary 1 of the first term is shifted to a value $\left|\Delta_{\max }^{r 1}\right|^{2}<1$ that decreases with increasing perturbation strength as is clearly visible in Fig. 8 The value $\left|\Delta_{\max }^{r 1}\right|^{2}$ provides a good estimate for the upper boundary of the overlap $|\Delta|^{2}$, although there are of course corrections from higher order approximations that smear the (total) overlap distribution $P\left(|\Delta|^{2}\right)$ about $\left|\Delta_{\max }^{r 1}\right|^{2}$, see for example Fig. 8 b).

\section{SUMMARY AND CONCLUSIONS}

In this paper we have studied the Anderson orthogonality catastrophe in generic mesoscopic systems with a finite number of electrons in discrete energy levels. Motivated by the 
study of mesoscopic photoabsorption spectra that will be the subject of the second paper II in this series, we have used the model of a rank one perturbation describing the perturbation left behind after the photoexcitation of a core electron into the conduction band. However, the basic underlying physics is much more general, and the model can be applied to any situation where a sudden, localized (rank one) perturbation acts on a finite number of chaotic electrons.

As a result of such a sudden perturbation, the overlap between the unperturbed and perturbed many-body ground states vanishes in the thermodynamic limit (AOC). In mesoscopic systems, even with few thousand electrons, this effect will be incomplete. Fluctuations cause a broad distribution of overlaps that is, however, bounded from above by a value smaller than 1 (for not too small perturbations) whereas there is a non-zero probability for having zero-overlap. The distribution of small overlaps is distinctly different for systems with or without time reversal symmetry (COE vs. CUE) and traces back to the difference in the Porter-Thomas distribution of their single-particle wavefunction intensities. The fluctuations originate from the levels around the Fermi energy (within a range smaller than the Thouless energy for systems we are interested in), and we derived an analytic expression for the lowest order (range 1) result that reproduces the distribution of small overlaps and is a good approximation for the whole distribution in case of strong perturbations.

\section{Acknowledgments}

We thank K. Matveev for several helpful discussions and I. Aleiner, Y. Gefen, I. Lerner, E. Mucciolo, and I. Smolyarenko for useful conversations. M. H. acknowledges the support of the Alexander von Humboldt Foundation. This work was supported in part by the NSF (DMR-0103003).
* Permanent address: Laboratoire de Physique Théorique et Modèles Statistiques (LPTMS), 91405 Orsay Cedex, France

II M. Hentschel, D. Ullmo, and H. U. Baranger, in preparation.

${ }^{1}$ For a comprehensive experimental review on the metallic case, see P. H. Citrin, G. K. Wertheim, and M. Schlüter, Phys. Rev. B 20, 3067 (1979) and references therein.

2 J. S. Lee, Y. Iwasa, and N. Miura, Semicond. Sci. Technol. 2, 675 (1987); M. S. Skolnick et al., Phys. Rev. Lett. 58, 2130 (1987).

3 B. Roulet, J. Gavoret, and P. Nozières, Phys. Rev. 178, 1072 (1969); P. Nozières, J. Gavoret, and B. Roulet, Phys. Rev. 178, 1084 (1969); P. Nozières and C. T. De Dominicis, Phys. Rev. 178, 1097 (1969).

${ }^{4}$ G. D. Mahan, Many-Particle Physics, 2nd edition, pp. 732 (Kluwer Academic/Plenum Publishers, New York, 1993).

5 K. D. Schotte and U. Schotte, Phys. Rev. 182, 479 (1969).

${ }^{6}$ K. Ohtaka and Y. Tanabe, Phys. Rev. B 28, 6833 (1983); Y. Tanabe and K. Ohtaka, Phys. Rev. B 29, 1653 (1984); K. Ohtaka and Y. Tanabe, Phys. Rev. B 30, 4235 (1984); Phys. Rev. B 34, 3717 (1986); Phys. Rev. B 39, 3054 (1989).

7 Theoretical work on the metallic x-ray edge problem is reviewed in K. Ohtaka and Y. Tanabe, Rev. Mod. Phys. 62, 929 (1990).

${ }^{8}$ M. Hentschel, D. Ullmo, and H. U. Baranger, Phys. Rev. Lett. 93, 176807 (2004).

9 L. L. Sohn, G. Schön, and L. P. Kouwenhowen, Mesoscopic Electron Transport (Kluwer, Dordrecht, 1997).

${ }^{10}$ C. W. J. Beenakker, Rev. Mod. Phys. 69, 731 (1997).

11 Y. Alhassid, Rev. Mod. Phys. 72, 895 (2000).

12 P. W. Anderson, Phys. Rev. Lett. 18, 1049 (1967).

13 R. L. Mössbauer and D. H. Sharp, Rev. Mod. Phys. 36, 410 (1964).

14 K. A. Matveev, L. I. Glazman, and H. U. Baranger, Phys. Rev. B 54, 5637 (1996).

15 D. A. Abanin and L. S. Levitov, Phys. Rev. Lett. 93, 126802 (2004).

16 K. A. Matveev and A. I. Larkin, Phys. Rev. B 46, 15337 (1992).
17 I. Hapke-Wurst, U. Zeitler, H. Frahm, A. G. M. Jansen, R. J. Haug, and K. Pierz, Phys. Rev. B. 62, 12621 (2000) and Refs. [5]-[7] therein.

18 J. M. Calleja, A. R. Goñi, B. S. Dennis, J. S. Weiner, A. Pinczuk, S. Schmitt-Rink, L. N. Pfeiffer, K. W. West, J. F. Müller, and A. E. Ruckenstein, Sol. St. Comm. 79, 911 (1991).

19 Y. Oreg and A. M. Finkelstein, Phys. Rev. B 53, 10928 (1996).

${ }^{20}$ Y. Chen and J. Kroha, Phys. Rev. B 46, 1332 (1992).

${ }^{21}$ I. L. Aleiner and K. A. Matveev, Phys. Rev. Lett. 80, 814 (1998).

${ }^{22}$ R. O. Vallejos, C. H. Lewenkopf, and Y. Gefen, Phys. Rev. B 65, 085309 (2002).

${ }^{23}$ Y. Gefen, R. Berkovits, I. V. Lerner, and B. L. Altshuler, Phys. Rev. B 65, 081106(R) (2002).

${ }^{24}$ O. Bohigas, in Chaos and Quantum Physics (Les Houches Session LII, 1989), edited by M.-J. Giannoni, A. Voros, and J. Zinn-Justin (North Holland, 1991), pp. 87.

25 M. L. Mehta, Random Matrices, 2nd edition (Academic Press, San Diego, 1991).

${ }^{26}$ I. E. Smolyarenko, F. M. Marchetti, and B. D. Simons, Phys. Rev. Lett. 88, 256808 (2002).

27 M. V. Berry, Proc. R. Soc. London 400, 229 (1985).

28 B. L. Altshuler and B. I. Shklovskii, Zh. Eksp. Teor. Fiz. 91, 220 (1986) [Sov. Phys. JETP 64, 127 (1986)].

29 W. H. Press, S. A. Teukolsky, W. T. Vetterling, B. P. Flannery, Numerical Recipes in C (Cambridge University Press, 1992), p. 623.

30 J. Friedel, Phil. Mag. 43, 153 (1952).

${ }^{31}$ M. Combescot and P. Nozières, J. Phys. 32, 913 (1971).

32 A. M. Zagoskin and I. Aflleck, J. Phys. A 30, 5743 (1997).

33 When the Metropolis algorithm is interrupted after a sufficient number of steps, the circle is "opened", and the runaway level is introduced by hand, neglecting possible fluctuations.

34 The range- 1 and range- 2 approximation in Fig. 8 are based on Eq. 35] and its generalization to include differences up to two mean level spacings $d$ on average for the range- 2 result. 